\title{
Síndrome de Wunderlich en una paciente con esclerosis tuberosa de Bourneville. Tratamiento conservador
}

\section{Wunderlich syndrome in tuberous sclerosis: Conservative management}

Héctor Iván Martínez-López, Fernando León-Vilchis, Luis Alfredo Jiménez-López, Abel Antonio RicardezEspinoza

\begin{abstract}
Resumen
ANTECEDENTES: El síndrome de Wunderlich, o hemorragia retroperitoneal espontánea, es una enfermedad hemorrágica renal poco frecuente. La etiología tumoral primaria del riñón, como el angiomiolipoma, representa $60 \%$ de los casos.

CASO CLíNICO: Paciente femenina de 22 años, con dolor de inicio súbito en la fosa renal derecha. $\mathrm{Al}$ ingreso hospitalario se encontraba consciente, orientada, con hipotensión leve; a la exploración física se observaron signos y síntomas de esclerosis tuberosa, abdomen blando, depresible, con dolor a la palpación y masa perceptible en la fosa renal derecha, sin datos de irritación peritoneal. La tomografía reportó un hematoma subcapsular derecho delimitado, con múltiples angiomiolipomas bilaterales. Se estableció el diagnóstico de síndrome de Wunderlich y la confirmación de esclerosis tuberosa por criterios mayores. Debido a la estabilidad hemodinámica se ofreció tratamiento conservador, vigilancia del hematocrito seriado, administración de líquidos intravenosos y reposo absoluto. La paciente evolucionó satisfactoriamente.

CONCLUSIÓN: El tratamiento de pacientes con síndrome de Wunderlich debe iniciar con la evaluación del estado hemodinámico y en caso de desequilibrio será necesaria la cirugía urgente. El caso expuesto en este estudio es una forma excepcional de síndrome de Wunderlich concomitante con esclerosis tuberosa y tratamiento conservador, que representa el primer reporte en la bibliografía médica con estas características.

PALABRAS CLAVE: Síndrome de Wunderlich; esclerosis tuberosa; hemorragia retroperitoneal espontánea.
\end{abstract}

Abstract

BACKGROUND: Wunderlich syndrome, also called spontaneous retroperitoneal hemorrhage, is a very rare clinical entity. Its primary kidney tumor etiology is angiomyolipoma and accounts for $60 \%$ of the cases.

CLINICAL CASE: A 22-year-old woman presented with sudden onset of pain in the right renal fossa. Upon hospital admission, the patient was conscious, lucid, and had mild hypotension. Physical examination revealed signs and symptoms of tuberous sclerosis, a soft, depressible abdomen that was painful upon palpation, a palpable mass in the right renal fossa, and no signs of peritoneal irritation. A tomography scan identified a right well-defined subcapsular hematoma with multiple bilateral angiomyolipomas. Wunderlich syndrome was diagnosed and tuberous sclerosis was confirmed through major criteria. Because the patient was hemodynamically stable she was managed conservatively, with serial hematocrit surveillance, intravenous fluids, and complete bedrest. The patient had satisfactory progression.

CONCLUSION: Treatment of Wunderlich syndrome should begin with an evaluation of the hemodynamic status of the patient. In case of instability, the indication is urgent surgery. We present herein the first report in the medical literature of an exceptional form of Wunderlich syndrome, concomitant with tuberous sclerosis, that was managed conservatively.

KEYWORDS: Wunderlich syndrome; Tuberous sclerosis; Spontaneous retroperitoneal hemorrhage.
Unidad Médica de Alta Especialidad, Centro Médico Nacional Lic. Adolfo Ruiz Cortines, Departamento de Urología, Hospital de Especialidades 14, Instituto Mexicano del Seguro Social, Veracruz, México.

Recibido: febrero 2018

Aceptado: julio 2018

Correspondencia Héctor Iván Martínez López hector.mtz.Ipz25@gmail.com

Este artículo debe citarse como Martínez-López HI, León-Vilchis F, Jiménez-López LA, Ricardez-Espinoza A. Síndrome de Wunderlich en una paciente con esclerosis tuberosa de Bourneville. Tratamiento conservador. Rev Mex Urol. 2018 julio-agosto;78(4): 303-309. DOI: https://doi.org/10.24245/revmexurol. v78i4.2019 


\section{ANTECEDENTES}

El síndrome de Wunderlich, o hemorragia retroperitoneal espontánea, es una colección hemática en la fosa renal, no relacionada con traumatismos. Esta alteración es poco frecuente, pero potencialmente mortal. Su origen se asocia con diversos mecanismos y representa una urgencia médica. ${ }^{1}$

El primer reporte de ruptura espontánea del parénquima renal fue descrito por Bonet en 1700; sin embargo, en 1856 Wunderlich informó un síndrome caracterizado por hemorragia perirrenal espontánea de localización intraperirrenal o subcapsular. ${ }^{1}$ El hemoperitoneo es un evento excepcional, pues la hemorragia está limitada por la resistencia del tejido perirrenal.

Los signos y síntomas suelen ser insidiosos y la tríada de Lenk (dolor lumbar intenso de inicio súbito, signos de hemorragia interna y tumoración retroperitoneal) sólo aparece en $20 \%$ de los casos.

La primera revisión del síndrome fue realizada por Polkey y Vynalek en 1933, quienes describieron las enfermedades del riñón como el principal factor asociado con hematoma retroperitoneal espontáneo no traumático. ${ }^{2}$

Las dos cohortes más grandes publicadas fueron las de Mc Dougal en 1975 (78 casos) y la Zhang y sus colaboradores en 2012 (un meta análisis con 165 casos de 47 publicaciones previas)..$^{3-4}$

El cuadro clínico del síndrome de Wunderlich es provocado por el sangrado de cualquier vaso u órgano del retroperitoneo, debido a enfermedades locales (riñón, glándula suprarrenal, anomalías vasculares) o sistémicas (periarteritis nodosa, coagulopatías, discrasias sanguíneas). ${ }^{5}$

Después de la rotura del aneurisma de aorta abdominal, las alteraciones renales representan las causas más frecuentes de hemorragia retroperitoneal espontánea. Los tumores siguen siendo responsables de $85 \%$ de los casos. ${ }^{6}$ Otras causas relacionadas con el síndrome de Wunderlich incluyen: pielonefritis, hidronefrosis, enfermedades vasculares renales $y$, en algunos casos, rechazo agudo o crónico de trasplante de riñón.?

El síndrome de Wunderlich de origen suprarrenal es menos frecuente y suele asociarse con situaciones de estrés, como sepsis, intervenciones quirúrgicas y pacientes quemados. ${ }^{8}$ También se ha reportado el tratamiento prolongado con corticoesteroides y corticotropina, feocromocitoma, adenoma, carcinoma, lesiones metastásicas y mielolipoma como causas adicionales del síndrome. El monitoreo de pacientes con síndrome de Wunderlich es importante para establecer el diagnóstico y tratamiento oportunos.

Los angiomiolipomas son tumores mesenquimatosos que frecuentemente forman micro o macroaneurismas, y provocan hemorragia espontánea. La manifestación de síntomas y el riesgo de sangrado es proporcional al tamaño de los angiomiolipomas. ${ }^{9}$

\section{CASO CLÍNICO}

Paciente femenina de 22 años que inició su padecimiento con dolor súbito en la fosa renal y lumbar derechas, con irradiación al flanco e hipocondrio de 30 horas de evolución. Al ingreso hospitalario se encontraba con hipotensión leve. Signos vitales: tensión arterial 90/60 mmHg, frecuencia cardiaca 28 por minuto, frecuencia respiratoria 25 por minuto y temperatura $36.7^{\circ} \mathrm{C}$. A la exploración física se encontró consciente y orientada, con regular estado de hidratación, leve palidez de tegumentos, angiofibromas faciales (Figura 1), placas fibrosas en las encías, múltiples depresiones (pits) en el esmalte dentario (Figura 2) y manchas hipocrómicas en el dorso (Figura 3); el sistema cardiorrespiratorio 


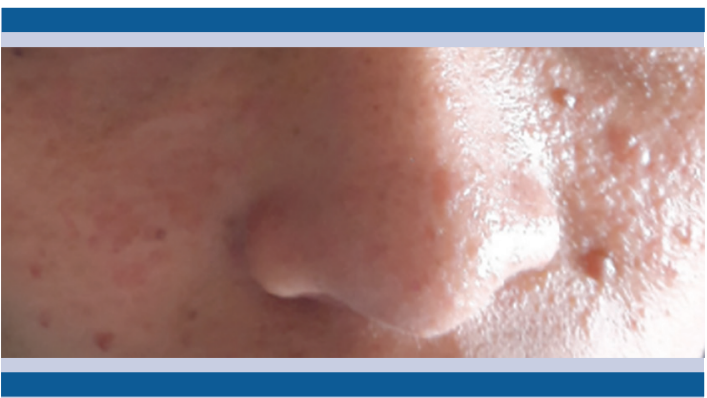

Figura 1. Múltiples angiofibromas faciales.

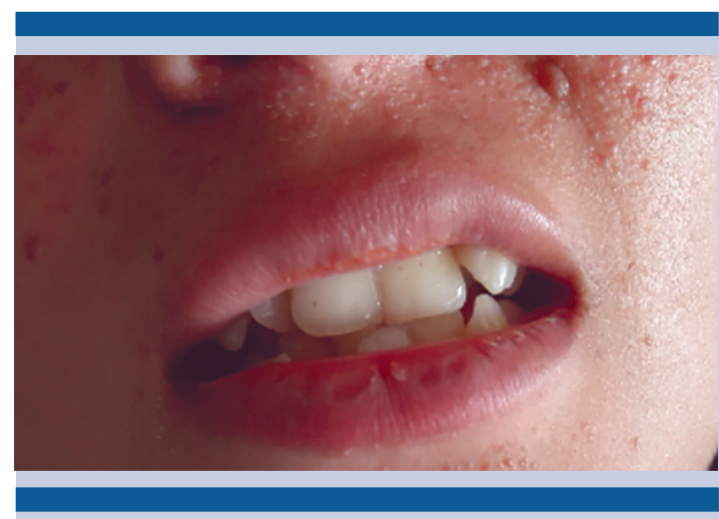

Figura 2. Depresiones (pits) en el esmalte dentario.

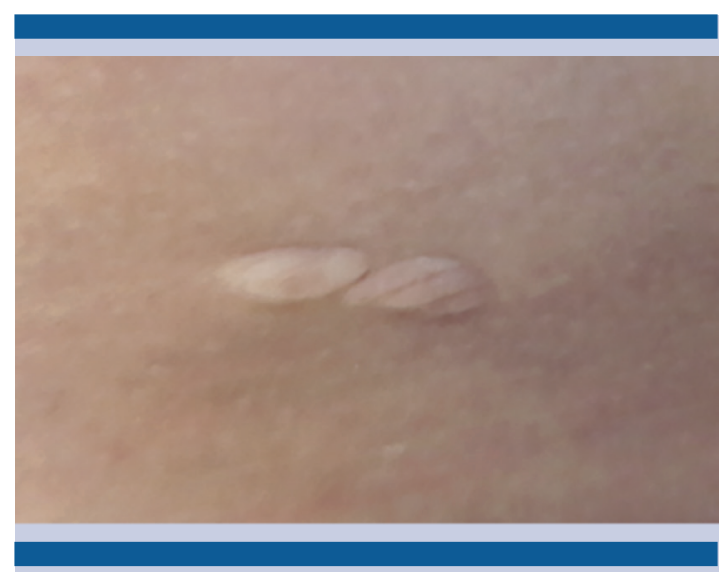

Figura 3. Manchas en hoja de serbal, hipocrómicas en el dorso.

sin alteraciones; abdomen blando, depresible, con dolor a la palpación en la fosa renal derecha y masa palpable en la fosa renal e hipocondrio derecho. No se apreciaron signos de irritación peritoneal, genitales de acuerdo con el sexo; extremidades íntegras, con buen Ilenado capilar y fibromas periungueales (tumores de Koenen) (Figura 4). Los estudios de laboratorio reportaron leucocitos: 12,000 mm33 $\mathrm{Hb}: 9.5 \mathrm{mg} / \mathrm{dL}$, HTO: $29 \%$ y Cr: $1.2 \mathrm{mg} / \mathrm{dL}$. Los tiempos de coagulación estuvieron dentro de los parámetros normales. El sedimento de orina con hematuria microscópica.

La tomografía evidenció un hematoma renal subscapular del lado derecho, con volumen aproximado de 80 cc, además de múltiples angiomiolipomas bilaterales de diferente tamaño (5-15 mm), sin líquido libre en la cavidad (Figura 5). Se estableció el diagnóstico de síndrome de Wunderlich y la confirmación de esclerosis tuberosa por criterios mayores. Debido a la estabilidad hemodinámica de la paciente, se ofreció tratamiento conservador, vigilancia del hematocrito seriado, administración de líquidos intravenosos y reposo absoluto. Cinco días después se otorgó el alta hospitalaria, sin alteraciones ni eventualidades adicionales. Actualmente se encuentra en buenas condiciones generales, en tratamiento con inhibidores de la mTOR (mammalian target of rapamycin) y más adelante se plantea la posibilidad de un trasplante renal.

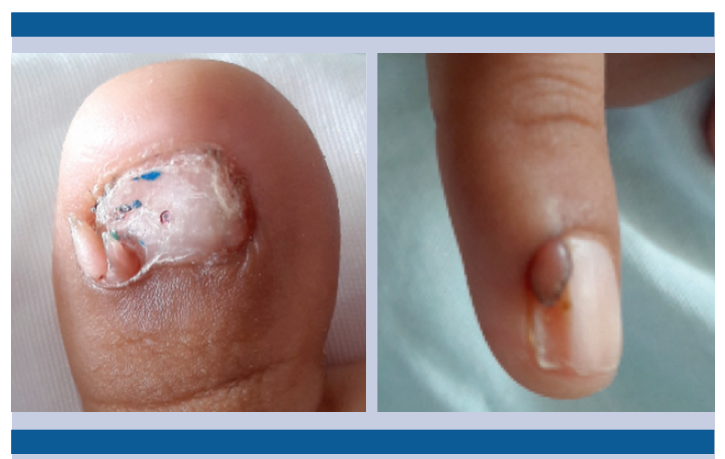

Figura 4. Fibromas periungueales o tumores de Koenen en las extremidades (patognomónico de la esclerosis tuberosa). 


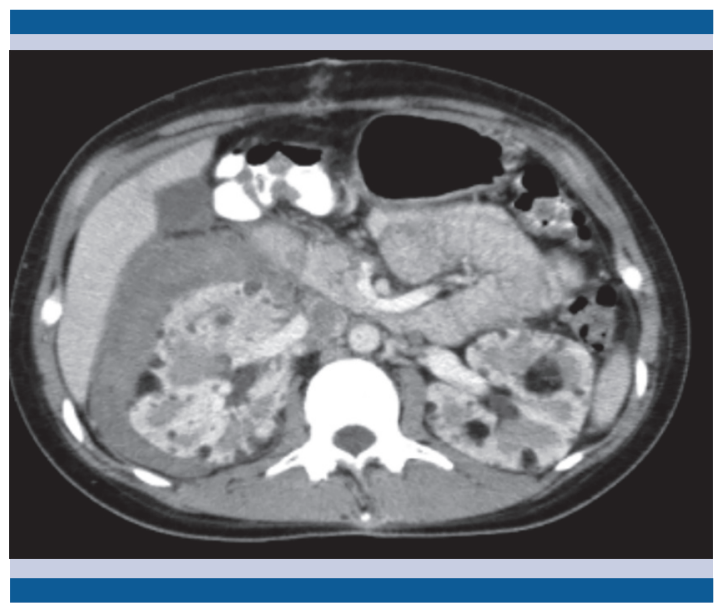

Figura 5. Tomografía abdominopélvica contrastada que evidencia un hematoma subcapsular del lado derecho, con múltiples angiomiolipomas bilaterales, sin líquido libre en la cavidad.

\section{DISCUSIÓN}

El síndrome de Wunderlich es un cuadro clínico definido, según la tríada de Lenk, por dolor lumbar de inicio súbito, rápida formación de un tumor palpable en el mismo sitio y choque hipovolémico. ${ }^{10,11}$ Algunos estudios señalan formas de manifestación insidiosa y progresiva, ${ }^{13}$ que resultan en sangrado lento o de escasa cuantía. Aunque se han descrito múltiples factores asociados con su origen, ${ }^{14}$ la mayor parte de las series indican al angiomiolipoma renal como la causa más frecuente. Este tumor representa una neoplasia benigna, histopatológicamente constituido por distintas proporciones de tejido adiposo, vascular y músculo liso. El 15\% de los tumores provoca sangrado espontáneo, sobre todo los mayores de $40 \mathrm{~mm}$, al parecer asociados con abundante y anormal coexistencia de estructuras vasculares deficientes de elastina, que favorecen la formación de aneurismas y estimulan el sangrado. El carcinoma renal de células claras concomitante con sangrado espontáneo varía de $0.3-1.3 \%$ de los casos (principalmente en lesiones de $1 \mathrm{a} 20 \mathrm{~cm}){ }^{4}$
En la actualidad, la mayor parte de los angiomiolipomas renales se detectan de forma fortuita, mediante estudios de imagen abdominales de rutina.

La tomografía computada es el método de elección para establecer el diagnóstico del sangrado retroperitoneal espontáneo, ${ }^{12}$ incluso para determinar su etiología, ${ }^{15}$ como sucedió en la paciente de este estudio, que permitió detectar el sangrado retroperitoneal que caracteriza el síndrome de Wunderlich y con ello planificar el tratamiento conservador.

La esclerosis tuberosa de Bourneville es una enfermedad genética de expresión clínica variable, caracterizada por la formación de tumores benignos denominados hamartomas. Estos tumores pueden aparecer en diversos órganos como: piel, sistema nervioso central, riñones, ojos, corazón y pulmones. ${ }^{16}$ La trasmisión es autosómica dominante, ligada a mutaciones de los genes TSC1 y TSC2 (tuberous sclerosis 1 y 2) que codifican a las proteínas hamartina y tuberina, respectivamente, para formar un complejo dimérico. ${ }^{17}$

La penetrancia de la enfermedad es de $100 \%$, pero las manifestaciones clínicas y su gravedad varían de una persona a otra, incluso entre parientes cercanos. ${ }^{18}$

Las manifestaciones renales más frecuentes incluyen angiomiolipomas y quistes renales. Los angiomiolipomas afectan entre 70 y $90 \%$ de los adultos con esclerosis tuberosa. En el caso aquí expuesto apareció en la adolescencia (promedio de edad de 11 años); suelen ser bilaterales, múltiples y de gran tamaño. Su incidencia aumenta con la edad y el crecimiento puede ser rápido ( $4 \mathrm{~cm}$ por año). ${ }^{19}$ Se han descrito casos en niños de corta edad, pero con baja incidencia (8-16\% antes de los 2 años). ${ }^{20}$ La lesión es común y más grave en mujeres. ${ }^{21}$ Desde el punto de vista histológico, se trata de hamartomas compues- 
tos por células musculares lisas, tejido adiposo y vasos sanguíneos anormales. No provocan síntomas y su detección es fortuita durante la ecografía abdominal. Los pacientes con tumores voluminosos (iguales o mayores a $4 \mathrm{~cm}$ ) suelen padecer hematuria, dolor abdominal, lumbalgia y expresar una masa palpable. ${ }^{22} \mathrm{La}$ complicación principal es la ruptura espontánea $y$, en consecuencia, un hematoma perirrenal o hemorragia retroperitoneal masiva, que puede provocar la muerte. El tratamiento de elección consiste en embolización; la cirugía se reserva para pacientes no aptos para esta última ${ }^{19}$ y el protocolo conservador se establece en sujetos con estabilidad hemodinámica. En pacientes con esclerosis tuberosa se estima un riesgo de hemorragia de $6 \%$ y aumenta con el tamaño de las lesiones. Los angiomiolipomas voluminosos o numerosos pueden provocar insuficiencia renal por destrucción parenquimatosa. ${ }^{19} \mathrm{El}$ diagnóstico se establece mediante ecografía abdominal; sin embargo, la tomografía computada o resonancia magnética son los estudios de elección. ${ }^{19}$

En pacientes con tumores de $3-4 \mathrm{~cm}$ puede proponerse tratamiento preventivo, es decir, embolización, exéresis o, de forma más reciente, prescripción de inhibidores de la vía mTOR. ${ }^{23} \mathrm{La}$ embolización representa el tratamiento de primera línea, pues reduce el tamaño de los tumores. Las complicaciones son mínimas, por ejemplo: síndrome posembolización, dolor y fiebre, que pueden controlarse con antiinflamatorios no esteroides o corticoesteroides por periodo corto. El riesgo de hemorragia espontánea después de la embolización es bajo (0-5.3\%), incluso se ha estimado que entre $6-50 \%$ de los casos pueden requerir una nueva embolización. ${ }^{19}$ La exéresis de los angiomiolipomas tiene menos recidivas, pero es más difícil de efectuar en pacientes con esclerosis tuberosa, debido al elevado número de lesiones. El tratamiento conservador implica la mayor preservación posible del tejido renal. Los estudios relacionados con los inhibidores de la vía mTOR han demostrado reducción de $50 \%$ del volumen de los angiomiolipomas; sin embargo, su efecto aún se discute (sirolimus), porque no se ha comprobado que la reducción del tumor disminuya el riesgo de hemorragia. ${ }^{23}$ Las complicaciones más frecuentes de los inhibidores de la vía mTOR incluyen: estomatitis, acné, diarrea, neumopatías, dislipidemias y amenorrea. Algunos autores los prescriben como primera línea de tratamiento en pacientes con angiomiolipomas asintomáticos mayores de $3 \mathrm{~cm},{ }^{24}$ y otros los indican sólo como segunda opción. ${ }^{19}$

Los quistes renales asociados con esclerosis tuberosa de Bourneville afectan entre 14 y $45 \%$ de los adultos y $10-20 \%$ de los niños. Suelen ser pequeños, simples, únicos o múltiples (menos de cinco) y asintomáticos. ${ }^{21}$ En casos excepcionales $(2 \%)$, la esclerosis tuberosa se relaciona desde la infancia con poliquistosis renal por pérdida. conjunta del gen $P K D 1$, que en la etapa adulta provoca insuficiencia renal crónica terminal.

Las lesiones cardiacas, cutáneas y cerebrales pueden aparecer en el lactante o el niño de corta edad, mientras que las renales y pulmonares se manifiestan de forma tardía. ${ }^{25}$ El diagnóstico se establece con base en criterios clínicos, radiológicos, histológicos y genéticos, estipulados en $1998{ }^{16}$ y actualizados en $2012 .{ }^{26}$ Los tres criterios clásicos (adenomas sebáceos, epilepsia, déficit intelectual) aparecen en $29 \%$ de los casos. ${ }^{27} \mathrm{El}$ tratamiento es complejo y requiere la participación de equipos multidisciplinarios.

El conocimiento de la fisiopatología de la vía mTOR (diana de rapamicina en células de mamífero) ha permitido la prescripción de este tipo de inhibidores en pacientes con enfermedades renales. ${ }^{23}$

\section{CONCLUSIONES}

El tratamiento del síndrome de Wunderlich se inicia con la evaluación del estado hemodiná- 
mico del paciente; en caso de desequilibrio o que no puede compensarse por otros métodos, está indicada la cirugía de urgencia. ${ }^{28}$ Casi siempre se consigue estabilizar al paciente para establecer el diagnóstico etiológico mediante exploraciones complementarias. Sin embargo, algunos autores proponen la nefrectomía radical, con previa comprobación de la integridad anatómica y funcional del riñón contralateral, siempre y cuando se haya descartado el origen traumático, por vasculitis o coagulopatía, con base en la alta incidencia de tumores malignos, como causa de la hemorragia renal espontánea y la posibilidad de adenocarcinomas periféricos de tamaño pequeño que escapan al diagnóstico de las exploraciones complementarias. ${ }^{29}$

En caso de contraindicación quirúrgica o ante la estabilidad hemodinámica del paciente, debe considerarse el tratamiento conservador, con seguimiento mediante tomografía abdominal seriada, con la finalidad de efectuar un estudio adecuado del origen de la hemorragia, antes de realizar una cirugía electiva resolutiva. ${ }^{30}$

Es importante conocer este síndrome, sus potenciales causas y la frecuencia relativa de cada una. La primera hipótesis diagnóstica debe ser la coexistencia de un tumor renal subyacente. La conducta se encamina al seguimiento clínico hasta la resolución del sangrado, incluso la postura más agresiva y necesaria, como la posibilidad de nefrectomía de urgencia, debido lesiones orgánicas que generan el sangrado. Debe valorarse de forma interdisciplinaria la conducta de cada paciente, además de considerar las limitaciones del medio hospitalario.

Se propone tratamiento conservador en pacientes estables hemodinámicamente; corrección del choque, la coagulopatía y embolización selectiva para las lesiones de origen vascular, angiomiolipomas $^{31}$ o lesiones bilaterales; sin embargo, en la mayoría de los casos, debido al desequilibrio hemodinámico y su origen, la nefrectomía re- presenta el tratamiento definitivo del síndrome. ${ }^{4}$ Hasta el momento, el tratamiento por vía laparoscópica no ha demostrado mejores resultados. ${ }^{32}$

El diagnóstico y tratamiento oportunos de ruptura renal espontánea es fundamental, por el riesgo que implica. El caso aquí expuesto es excepcional, pues la evolución del síndrome fue progresiva, dando la oportunidad de estudiarlo, incluso evaluar la tomografía de cráneo, donde se evidenciaron los nódulos subependimarios y las tuberosidades corticales asintomáticas (Figura 6), para después establecer el tratamiento conservador, debido a la estabilidad hemodinámica de la paciente.

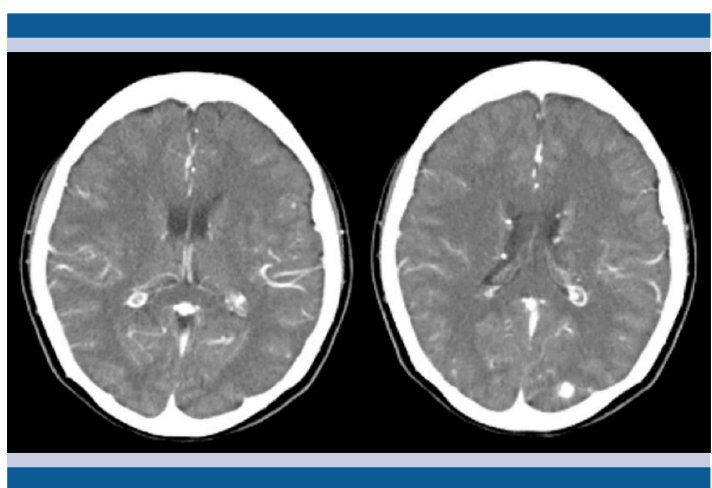

Figura 6. Tomografía de cráneo contrastada que muestra nódulos subependimarios y tuberosidades corticales.

De acuerdo con la bibliografía consultada, no existe ningún caso de síndrome de Wunderlich causado por angiomiolipomas en pacientes con esclerosis tuberosa tratados de forma conservadora. El tratamiento del síndrome depende, fundamentalmente, de dos factores: 1) estado hemodinámico del paciente y 2) origen del sangrado. Se sugiere una conducta conservadora inicial y el tratamiento quirúrgico se reserva en caso de descompensación hemodinámica.

Nuestra paciente se encuentra en tratamiento con inhibidores de la mTOR, con posibilidad de trasplante renal a futuro. 


\section{REFERENCIAS}

1. Wunderlich. Handbuch der patholie und therapie. $2^{\text {nd }}$ ed. Stuttgart: Ebner and Seubert, 1856.

2. Polkey and Vynalek. Spontaneous nontraumatic perirrenal and renal hematomas. An experimental and clinical study. Arch Surg 1993;26:196

3. McDougal W, Kursh E, Persky L. Spontaneous rupture of kidney with perirrenal haematoma. J Urol 1975;114:181-4.

4. Zhang JQ, Fielding JR, Zou KH. Etiology of spontaneous perirenal hemorrhage: a meta-analysis. J Urol 2002;167(4):1593-6.

5. Pove D, Caine M. Spontaneous retroperitoneal hemorrhage. J Urol 1992;147:311-8.

6. Stewart B, McLaughlin SJ, Thompson GA. Spontaneous retroperitoneal hemorrhage: a general surgeon's perspective. Aust N Z J Surg 1998;68(5):371-3.

7. Pinilla R, López S, Quintana JC, Al-Ezzi Al-Malahi A. Síndrome de Wunderlich: presentación de un caso y revisión de la bibliografía. Rev Colomb Cir 2009;24:56-61.

8. Daliakopoulus SI. Spontaneous retroperitoneal hematoma: a rare devastating clinical entity of a pleiada of less common origins. doi: 10.4103/2006-8808.78462.

9. De Luca S, Terrone C, Rossetti SR. Management of renal angiomyolipoma: a report of 53 cases. BJU Int 1999;83(3):215-8.

10. Machuca Santa-Cruz FJ, Barros Rodriguez JM, Guate Ortiz JL, Mata Varela J, Ojea Calvo A, Figueiredo Godoy L, et al. Cirugía conservadora de urgencia por rotura de angiomiolipoma renal. Arch Esp Urol 1990;43:179-81.

11. Pode D, Metetik S, Shapiro A, Lame M, Frcs MS. Diagnosis and management of renal angiomiolipoma. Urology 1985;5:461-7.

12. Gimeno-Argente $V$, Bosquet-Sanz M, Ramírez-Backhaus M, Trassierra-Villa M, Arlandis-Guzmán S, Jiménez-Cruz JF. Hemorragia retroperitoneal espontánea: nuestra experiencia en los últimos 10 años. Actas Urol Esp 2007;31:521-7.

13. López-Cubillana $P$, Hita-Rosino E, Asensio Egea L, Rigabert Montiel M, Hita Villaplana G, Pérez Albacete M. Síndrome de Wünderlich. Revisión diagnóstica y terapéutica. A propósito de siete observaciones. Actas Urol Esp 1995;19:772-6.

14. Alonso A, Domínguez F, Verez M, Rodríguez B, Benavente J, Barros JM, et al. Síndrome de Wünderlich por metástasis de sarcoma gástrico en riñón. Aportación de un caso. Actas Urol Esp 1996;20:395-8.

15. Albi G, Del Campo L, Tagarro D. Wünderlich's syndrome: causes, diagnosis and radiological management. Clin Radiol 2002;57:840-5.

16. Roach ES, Gomez MR, Northrup H. Tuberous sclerosis complex consensus conference: revised clinical diagnostic criteria. J Child Neurol 1998;13:624-8.

17. European Chromosome 16 Tuberous Sclerosis Consortium. Identification and characterization of the tuberous sclerosis gene on chromosome 16. Cell 1993;75:1305-15.
18. Northrup H, Koenig MK, Au KS. Tuberous sclerosis complex. In: Adam MP, Ardinger HH, Pagon RA, Wallace SE, et al., editores. GeneReviews ${ }^{\circledR}$ [Internet]. Seattle (WA): University of Washington, Seattle; 1993-2014, 13 juillet 1999 [mise à jour 23 novembre 2011].

19. Rouvière $\mathrm{O}$, Nivet $\mathrm{H}$, Grenier N, Zini L, Lechevallier E. Kidney damage due to tuberous sclerosis complex: management recommendations. Prog Urol 2012; 22:367-79.

20. Jozwiak S, Schwartz RA, Janniger CK, Bielicka-Cymerman J. Usefulness of diagnostic criteria of tuberous sclerosis complex in pediatric patients. J Child Neurol 2000;15:652-9.

21. Rakowski SK, Winterkorn EB, Paul E, Steele DJ, et al. Renal manifestations of tuberous sclerosis complex: Incidence, prognosis, and predictive factors. Kidney Int 2006;70:1777-82.

22. Ballanger F, Quéreux G, Stalder JF, Schmitt S, Jacquemont S. Sclérose tubéreuse de Bourneville. EMC (Elsevier Masson SAS, Paris), Dermatologie, 98-760-A-10, 2005:8.

23. Bissler JJ,. Sirolimus for angiomyolipoma in tuberous sclerosis complex or lymphangioleiomyomatosis. doi: 10.1056/NEJMoa063564.

24. Krueger DA, Northrup H, International Tuberous Sclerosis Complex Consensus Group. Tuberous sclerosis complex surveillance and management: recommendations of the 2012 International Tuberous Sclerosis Complex Consensus Conference. Pediatr Neurol 2013;49:255-65.

25. Serra A, Bonny O, Bürki S, Dorn T, Fuster D, Guzman R, et al. La sclérose tubéreuse de Bourneville: pathogenèse, clinique et nouvelles options thérapeutiques. Forum Med Suisse 2013;13:696-702.

26. Northrup $\mathrm{H}$, et al. Tuberous sclerosis complex diagnostic criteria update: recommendations of the 2012 international tuberous sclerosis complex consensus conference. doi: 10.1016/j.pediatrneurol.2013.08.001

27. Schwartz RA, Fernández G, Kotulska K, Józwiak S. Tuberous sclerosis complex: advances in diagnosis, genetics, and management. J Am Acad Dermatol 2007;57:189-202.

28. Grasso M, Blanco S, Fortuna F, Crippa S, Di Bella C. Spontaneous rupture of renal leiomyosarcoma in a 45-year-old woman. Arch Esp Urol 2004;57:870-2.

29. Hao LW, Lin CM, Tsai SH. Spontaneous hemorrhagic angiomyolipoma presents with massive hematuria leading to urgent nephrectomy. Am J Emerg Med. 2008;26:249e3-5.

30. Sales R, Villa V, Caballé J, Mas A, Valencoso O, Ballús L, et al. Síndrome de Wünderlich. Hemorragia renal espontánea. Cir Esp 2000;68:493-5.

31. Moratalla MB. Wunderlich's syndrome due to spontaneous rupture of large bilateral angiomyolipomas. doi: 10.1136/ emj.2008.062091.

32. Hernandez F, Ong AM, Rha KH, Pinto PA, Kavoussi LR. Laparoscopic renal surgery after spontaneous retroperitoneal hemorrhage. J Urol 2003;170(3):749-51. 